\title{
COMPOSITE FUNCTIONS WITH ALLEN DETERMINANTS AND THEIR APPLICATIONS TO PRODUCTION MODELS IN ECONOMICS
}

\author{
M. EVREN AYDIN AND MAHMUT ERGUT
}

\begin{abstract}
In this paper, we derive an explicit formula for the Allen determinants of composite functions of the form:

$$
f(\mathbf{x})=F\left(h_{1}\left(x_{1}\right) \times \cdots \times h_{n}\left(x_{n}\right)\right) .
$$

We completely classify the composite functions by using their Allen determinants. Some applications of Allen determinants to production models are also given.
\end{abstract}

\section{Introduction}

In economics, a production function is a mathematical expression which denotes the physical relations between the output generated of a firm, an industry or an economy and inputs that have been used. Explicitly, a production function is a map which has non-vanishing first derivatives defined by

$$
f: \mathbb{R}_{+}^{n} \longrightarrow \mathbb{R}_{+}, f=f\left(x_{1}, \ldots, x_{n}\right),
$$

where $f$ is the quantity of output, $n$ are the number of inputs and $x_{1}, \ldots, x_{n}$ are the inputs. The production functions satisfy the following conditions:

1. $f$ is equivalently zero in absence of an input.

2. $\frac{\partial f}{\partial x_{i}}>0$, for $i=1, \ldots, n$, which means that the production function is strictly increasing with respect to any factor of production.

3. $\frac{\partial^{2} f}{\partial x_{i}^{2}}<0$, for $i=1, \ldots, n$, i.e., the production has decrasing efficiency with respect to any factor of production.

4. $f(\mathbf{x}+\mathbf{y})=f(\mathbf{x})+f(\mathbf{y}), \forall \mathbf{x}, \mathbf{y} \in \mathbb{R}_{+}^{n}$, which implies that the production has non-decreasing global efficiency. For more detailed properties of production functions, see $[2,13,15,17$, $19,21,22]$.

Received January 1, 2014, accepted March 3, 2014.

2010 Mathematics Subject Classification. 91B38, 15A15, 53B25.

Key words and phrases. Production function, generalized Cobb-Douglas production function, composite function, Allen elasticity of substitution.

Corresponding author: M. Evren Aydin. 
A production function $f\left(x_{1}, \ldots, x_{n}\right)$ is said to be homogeneous of degree $p$ or $p$-homogenous if

$$
f\left(t x_{1}, \ldots, t x_{n}\right)=t^{p} f\left(x_{1}, \ldots, x_{n}\right)
$$

holds for each $t \in \mathbb{R}_{+}$for which (1.1) is defined. A homogeneous function of degree one is called linearly homogeneous. Many important properties of homogeneous production functions in economics were interpreted in terms of the geometry of their graphs by $[4,5,11,17$, 18].

A. D. Vilcu and G. E. Vilcu [23] gave an exact classification for homogeneous production functions with proportional marginal rate of substitution and with constant elasticity of labor and capital.

The most common quantitative indices of production factor substitutability are forms of the elasticity of substitution. R.G.D. Allen and J.R. Hicks [1] suggested two generalizations of Hicks' original two variable elasticity concept.

The first concept, called Hicks elasticity of substitution, is defined as follows.

Let $f\left(x_{1}, \ldots, x_{n}\right)$ be a production function. Then Hicks elasticity of substitution of the $i$-th production variable with respect to the $j$-th production variable is given by

$$
H_{i j}(\mathbf{x})=-\frac{\frac{1}{x_{i} f_{i}}+\frac{1}{x_{j} f_{j}}}{\frac{f_{i i}}{\left(f_{i}\right)^{2}}-\frac{2 f_{i j}}{f_{i} f_{j}}+\frac{f_{j j}}{\left(f_{j}\right)^{2}}}\left(\mathbf{x} \in \mathbb{R}_{+}^{n}, i, j=1, \ldots, n, i \neq j\right),
$$

where $f_{i}=\partial f / \partial x_{i}, f_{i j}=\partial^{2} f / \partial x_{i} \partial x_{j}$.

L. Losonczi [16] classified homogeneous production functions of 2 variables, having constant Hicks elasticiy of substitution. Then, the classification of L. Losonczi was extended to $n$ variables by B-Y. Chen [6].

The second concept, investigated by R.G.D. Allen and H. Uzawa [20], is the following:

Let $f$ be a production function. Then Allen elasticity of substitution of the $i$-th production variable with respect to the $j$-th production variable is defined by

$$
A_{i j}(\mathbf{x})=-\frac{x_{1} f_{1}+x_{2} f_{2}+\cdots+x_{n} f_{n}}{x_{i} x_{j}} \frac{D_{i j}}{D}\left(\mathbf{x}=\left(x_{1}, \ldots, x_{n}\right) \in \mathbb{R}_{+}^{n}, i, j=1, \ldots, n, i \neq j\right),
$$

where $D$ is the determinant of the matrix

$$
M(f)=\left(\begin{array}{cccc}
0 & f_{1} & \ldots & f_{n} \\
f_{1} & f_{11} & \ldots & f_{1 n} \\
\vdots & \vdots & \ldots & \vdots \\
f_{n} & f_{n 1} & \ldots & f_{n n}
\end{array}\right)
$$


and $D_{i j}$ is the co-factor of the element $f_{i j}$ in the determinant $D(D \neq 0$ is assumed). $M(f)$ is called the Allen's matrix and we call $\operatorname{det}(M(f))$ the Allen determinant.

It is a simple calculation to show that in case of two variables Hicks elasticity of substitution coincides with Allen elasticity of substitution.

In this paper, by analogy to the Hessian Determinant Formula given by B.-Y. Chen in [10] for composite functions of the form $f(\mathbf{x})=F\left(h_{1}\left(x_{1}\right)+\cdots+h_{n}\left(x_{n}\right)\right)$, we give an explicit formula for Allen determinant of the composite functions of the form

$$
f(\mathbf{x})=F\left(h_{1}\left(x_{1}\right) \times \cdots \times h_{n}\left(x_{n}\right)\right) .
$$

We classify the composite functions given by (1.3) using their Allen determinants. Some geometric applications of Allen determinants are also given.

\section{Allen determinant formula and classification of composite functions}

Throughout this article, we assume that $h_{1}, \ldots, h_{n}: \mathbb{R} \longrightarrow \mathbb{R}$ are thrice differentiable functions and $F: I \subset \mathbb{R} \longrightarrow \mathbb{R}$ is a twice differentiable function with $F^{\prime}(u) \neq 0$ such that $I \subset \mathbb{R}$ is an interval of positive length.

The following provides an explicit formula for the Allen determinant of a composite function given by (1.3).

Allen Determinant Formula. The determinant of the Allen matrix $M(f)$ of the composite function $f=F\left(h_{1}\left(x_{1}\right) \times \cdots \times h_{n}\left(x_{n}\right)\right)$ is given by

$$
\operatorname{det}(M(f))=-u^{n+1}\left(F^{\prime}\right)^{n+1} \sum_{j=1}^{n}\left(\frac{h_{1}^{\prime}}{h_{1}}\right)^{\prime} \ldots\left(\frac{h_{j-1}^{\prime}}{h_{j-1}}\right)^{\prime}\left(\frac{h_{j}^{\prime}}{h_{j}}\right)^{2}\left(\frac{h_{j+1}^{\prime}}{h_{j+1}}\right)^{\prime} \ldots\left(\frac{h_{n}^{\prime}}{h_{n}}\right)^{\prime},
$$

where $h_{j}^{\prime}=\frac{d h_{j}}{d x_{j}}$ and $F^{\prime}=F^{\prime}(u)$ for $u=h_{1}\left(x_{1}\right) \times \cdots \times h_{n}\left(x_{n}\right)$.

Proof. Let $f$ be a twice differentiable composite function given by

$$
f(\mathbf{x})=F\left(h_{1}\left(x_{1}\right) \times \cdots \times h_{n}\left(x_{n}\right)\right),
$$

where $\mathbf{x}=\left(x_{1}, \ldots, x_{n}\right) \in \mathbb{R}^{n}$. It follows from (2.2) that

$$
f_{i}=\frac{\partial f}{\partial x_{i}}=\frac{h_{i}^{\prime}}{h_{i}} u F^{\prime}, f_{i j}=\frac{\partial^{2} f}{\partial x_{i} \partial x_{j}}=\frac{h_{i}^{\prime} h_{j}^{\prime}}{h_{i} h_{j}} u\left[F^{\prime}+u F^{\prime \prime}\right], 1 \leq i \neq j \leq n,
$$

and

$$
f_{i i}=\frac{\partial^{2} f}{\partial x_{i}^{2}}=\frac{h_{i}^{\prime \prime}}{h_{i}} u F^{\prime}+\left(\frac{h_{i}^{\prime}}{h_{i}}\right)^{2} u^{2} F^{\prime \prime}
$$


By using (2.3) and (2.4), the determinant of Allen matrix $M(f)$ of the composite function given by (2.2) is

$$
\operatorname{det}(M(f))=\left|\begin{array}{ccccc}
0 & \frac{h_{1}^{\prime}}{h_{1}} u F^{\prime} & \frac{h_{2}^{\prime}}{h_{2}} u F^{\prime} & \ldots & \frac{h_{n}^{\prime}}{h_{n}} u F^{\prime} \\
\frac{h_{1}^{\prime}}{h_{1}} u F^{\prime} & \frac{h_{1}^{\prime \prime}}{h_{1}} u F^{\prime}+\left(\frac{h_{1}^{\prime}}{h_{1}}\right)^{2} u^{2} F^{\prime \prime} & \frac{h_{1}^{\prime} h_{2}^{\prime}}{h_{1} h_{2}} u\left[F^{\prime}+u F^{\prime \prime}\right] & \ldots & \frac{h_{1}^{\prime} h_{n}^{\prime}}{h_{1} h_{n}} u\left[F^{\prime}+u F^{\prime \prime}\right] \\
\frac{h_{2}^{\prime}}{h_{2}} u F^{\prime} & \frac{h_{1}^{\prime} h_{2}^{\prime}}{h_{1} h_{2}} u\left[F^{\prime}+u F^{\prime \prime}\right] & \frac{h_{2}^{\prime \prime}}{h_{2}} u F^{\prime}+\left(\frac{h_{2}^{\prime}}{h_{2}}\right)^{2} u^{2} F^{\prime \prime} & \ldots & \frac{h_{2}^{\prime} h_{n}^{\prime}}{h_{2} h_{n}} u\left[F^{\prime}+u F^{\prime \prime}\right] \\
\vdots & \vdots & \vdots & \ldots & \vdots \\
\frac{h_{n}^{\prime}}{h_{n}} u F^{\prime} & \frac{h_{1}^{\prime} h_{n}^{\prime}}{h_{1} h_{n}} u\left[F^{\prime}+u F^{\prime \prime}\right] & \frac{h_{2}^{\prime} h_{n}^{\prime}}{h_{2} h_{n}} u\left[F^{\prime}+u F^{\prime \prime}\right] & \ldots & \frac{h_{n}^{\prime \prime}}{h_{n}} u F^{\prime}+\left(\frac{h_{n}^{\prime}}{h_{n}}\right)^{2} u^{2} F^{\prime \prime}
\end{array}\right|
$$

Now we apply Gauss elimination method for the determinant from the formula (2.5). We replace the second column by second column minus $\frac{h_{1}^{\prime}}{h_{1}}\left(1+\frac{u \mathrm{~F}^{\prime \prime}}{F^{\prime}}\right)$ times the first column; then we derive

$$
\operatorname{det}(M(f))=\left|\begin{array}{ccccc}
0 & \frac{h_{1}^{\prime}}{h_{1}} u F^{\prime} & \frac{h_{2}^{\prime}}{h_{2}} u F^{\prime} & \cdots & \frac{h_{n}^{\prime}}{h_{n}} u F^{\prime} \\
\frac{h_{1}^{\prime}}{h_{1}} u F^{\prime}\left(\frac{h_{1}^{\prime}}{h_{1}}\right)^{\prime} u F^{\prime} & \frac{h_{1}^{\prime} h_{2}^{\prime}}{h_{1} h_{2}} u\left[F^{\prime}+u F^{\prime \prime}\right] & \cdots & \frac{h_{1}^{\prime} h_{n}^{\prime}}{h_{1} h_{n}} u\left[F^{\prime}+u F^{\prime \prime}\right] \\
\frac{h_{2}^{\prime}}{h_{2}} u F^{\prime} & 0 & \frac{h_{2}^{\prime \prime}}{h_{2}} u F^{\prime}+\left(\frac{h_{2}^{\prime}}{h_{2}}\right)^{2} u^{2} F^{\prime \prime} & \cdots & \frac{h_{2}^{\prime} h_{n}^{\prime}}{h_{2} h_{n}} u\left[F^{\prime}+u F^{\prime \prime}\right] \\
\vdots & \vdots & \vdots & \cdots & \vdots \\
\frac{h_{n}^{\prime}}{h_{n}} u F^{\prime} & 0 & \frac{h_{2}^{\prime} h_{n}^{\prime}}{h_{2} h_{n}} u\left[F^{\prime}+u F^{\prime \prime}\right] & \cdots & \frac{h_{n}^{\prime \prime}}{h_{n}} u F^{\prime}+\left(\frac{h_{n}^{\prime}}{h_{n}}\right)^{2} u^{2} F^{\prime \prime}
\end{array}\right| .
$$

By similar elementary transformations, we get

$$
\operatorname{det}(M(f))=\left|\begin{array}{ccccc}
0 & \frac{h_{1}^{\prime}}{h_{1}} u F^{\prime} & \frac{h_{2}^{\prime}}{h_{2}} u F^{\prime} & \ldots & \frac{h_{n}^{\prime}}{h_{n}} u F^{\prime} \\
\frac{h_{1}^{\prime}}{h_{1}} u F^{\prime}\left(\frac{h_{1}^{\prime}}{h_{1}}\right)^{\prime} u F^{\prime} & 0 & \ldots & 0 \\
\frac{h_{2}^{\prime}}{h_{2}} u F^{\prime} & 0 & \left(\frac{h_{2}^{\prime}}{h_{2}}\right)^{\prime} u F^{\prime} & \ldots & 0 \\
\vdots & \vdots & \vdots & \ldots & \vdots \\
\frac{h_{n}^{\prime}}{h_{n}} u F^{\prime} & 0 & 0 & \ldots\left(\frac{h_{n}^{\prime}}{h_{n}}\right)^{\prime} u F^{\prime}
\end{array}\right|
$$

After calculating the determinant from the formula (2.6), we finally obtain

$$
\operatorname{det}(M(f))=-u^{n+1}\left(F^{\prime}\right)^{n+1} \sum_{j=1}^{n}\left(\frac{h_{1}^{\prime}}{h_{1}}\right)^{\prime} \ldots\left(\frac{h_{j-1}^{\prime}}{h_{j-1}}\right)^{\prime}\left(\frac{h_{j}^{\prime}}{h_{j}}\right)^{2}\left(\frac{h_{j+1}^{\prime}}{h_{j+1}}\right)^{\prime} \ldots\left(\frac{h_{n}^{\prime}}{h_{n}}\right)^{\prime},
$$

which completes the proof.

Since it is assumed that $F^{\prime} \neq 0$, the Allen Determinant Formula follows immediately

Corollary 2.1. Let $f=F\left(h_{1}\left(x_{1}\right) \times \cdots \times h_{n}\left(x_{n}\right)\right)$ be a twice differentiable composite function. Then the singularity of Allen's matrix of $f$ only depends on the functions $h_{1}, \ldots, h_{n}$. 
Next result completely classifies the composite functions in the form

$$
f=F\left(h_{1}\left(x_{1}\right) \times \cdots \times h_{n}\left(x_{n}\right)\right)
$$

whose Allen's matrices are singular.

Theorem 2.2. Let $F(u)$ be a twice differentiable function with $F^{\prime}(u) \neq 0$ and let $f$ be a composite function given by

$$
f=F\left(h_{1}\left(x_{1}\right) \times \cdots \times h_{n}\left(x_{n}\right)\right),
$$

where $h_{1}, \ldots, h_{n}$ are thrice differentiable and nonzero functions. Then the Allen matrix $M(f)$ of $f$ is singular if and only if $f$ is one of the following:

(i) $f=F\left(\gamma e^{\alpha_{1} x_{1}+\alpha_{2} x_{2}} \times h_{3}\left(x_{3}\right) \times \cdots \times h_{n}\left(x_{n}\right)\right)$, where $\gamma, \alpha_{1}, \alpha_{2}$ are nonzero constants;

(ii) $f=F\left(\gamma\left(x_{1}+\beta_{1}\right)^{\alpha_{1}} \times \cdots \times\left(x_{n}+\beta_{n}\right)^{\alpha_{n}}\right)$, where $\gamma, \alpha_{i}$ are nonzero constants satisfying $\alpha_{1}+$ $\cdots+\alpha_{n}=0$ and $\beta_{i}$ some constants.

Proof. Let $f=F\left(h_{1}\left(x_{1}\right) \times \cdots \times h_{n}\left(x_{n}\right)\right)$ be a twice differentiable composite function. Let us assume that the Allen matrix $M(f)$ of $f$ is singular. Then, by Allen Determinant formula, we get

$$
0=\operatorname{det}(M(f))=-u^{n+1}\left(F^{\prime}\right)^{n+1} \sum_{j=1}^{n}\left(\frac{h_{1}^{\prime}}{h_{1}}\right)^{\prime} \ldots\left(\frac{h_{j-1}^{\prime}}{h_{j-1}}\right)^{\prime}\left(\frac{h_{j}^{\prime}}{h_{j}}\right)^{2}\left(\frac{h_{j+1}^{\prime}}{h_{j+1}}\right)^{\prime} \ldots\left(\frac{h_{n}^{\prime}}{h_{n}}\right)^{\prime},
$$

where $u=h_{1}\left(x_{1}\right) \times \cdots \times h_{n}\left(x_{n}\right)$. Because of $F^{\prime}(u) \neq 0$, the equation (2.7) takes the form:

$$
\sum_{j=1}^{n}\left(\frac{h_{1}^{\prime}}{h_{1}}\right)^{\prime} \ldots\left(\frac{h_{j-1}^{\prime}}{h_{j-1}}\right)^{\prime}\left(\frac{h_{j}^{\prime}}{h_{j}}\right)^{2}\left(\frac{h_{j+1}^{\prime}}{h_{j+1}}\right)^{\prime} \ldots\left(\frac{h_{n}^{\prime}}{h_{n}}\right)^{\prime}=0 .
$$

Case (i): At least one of $\left(\frac{h_{1}^{\prime}}{h_{1}}\right)^{\prime}, \ldots,\left(\frac{h_{n}^{\prime}}{h_{n}}\right)^{\prime}$ vanishes. Without loss of generality, we may assume that $\left(\frac{h_{1}^{\prime}}{h_{1}}\right)^{\prime}=0$. Hence from $(2.8)$ we get

$$
\left(\frac{h_{1}^{\prime}}{h_{1}}\right)^{2}\left(\frac{h_{2}^{\prime}}{h_{2}}\right)^{\prime}\left(\frac{h_{3}^{\prime}}{h_{3}}\right)^{\prime} \ldots\left(\frac{h_{n}^{\prime}}{h_{n}}\right)^{\prime}=0
$$

Without loss of generality, we may assume from (2.9) that $\left(\frac{h_{2}^{\prime}}{h_{2}}\right)^{\prime}=0$. Thus we have

$$
h_{1}\left(x_{1}\right)=\gamma_{1} e^{\alpha_{1} x_{1}}, h_{2}\left(x_{2}\right)=\gamma_{2} e^{\alpha_{2} x_{2}},
$$

for some nonzero constants $\alpha_{1}, \alpha_{2}, \gamma_{1}, \gamma_{2}$. This gives case (i) of the theorem.

Case (ii): $\left(\frac{h_{1}^{\prime}}{h_{1}}\right)^{\prime}, \ldots,\left(\frac{h_{n}^{\prime}}{h_{n}}\right)^{\prime}$ are nonzero. Then from (2.8), by dividing with the product $\left(\frac{h_{1}^{\prime}}{h_{1}}\right)^{\prime} \ldots\left(\frac{h_{n}^{\prime}}{h_{n}}\right)^{\prime}$, we write

$$
\frac{\left(\frac{h_{1}^{\prime}}{h_{1}}\right)^{2}}{\left(\frac{h_{1}^{\prime}}{h_{1}}\right)^{\prime}}+\cdots+\frac{\left(\frac{h_{n}^{\prime}}{h_{n}}\right)^{2}}{\left(\frac{h_{n}^{\prime}}{h_{n}}\right)^{\prime}}=0 .
$$


Taking the partial derivative of (2.10) with respect to $x_{i}$, we obtain

$$
2-\left(\frac{h_{i}^{\prime}}{h_{i}}\right) \frac{\left(\frac{h_{i}^{\prime}}{h_{i}}\right)^{\prime \prime}}{\left[\left(\frac{h_{i}^{\prime}}{h_{i}}\right)^{\prime}\right]^{2}}=0 .
$$

By solving (2.11), we find

$$
h_{i}=\gamma_{i}\left(x_{i}+\beta_{i}\right)^{\alpha_{i}}
$$

for some nonzero constants $\gamma_{i}, \alpha_{i}$ satisfying $\alpha_{1}+\cdots+\alpha_{n}=0$ and some constants $\beta_{i}$. Therefore, we complete first part of the proof.

The converse can be verified directly.

\section{Some applications of Allen determinants to production models}

In economics, goods that are completely substitutable with each other are called perfect substitutes. Mathematically, a production function is a perfect substitute if it is of the form:

$$
f\left(x_{1}, \ldots, x_{n}\right)=\sum_{i=1}^{n} a_{i} x_{i},
$$

where $a_{1}, \ldots, a_{n}$ are nonzero constants [12].

In 1928, C. W. Cobb and P. H. Douglas introduces in [13] a famous two-factor production function

$$
Y=b L^{k} C^{1-k}
$$

where $b$ presents the total factor productivity, $Y$ the total production, $L$ the labor input and $C$ the capital input. This function is nowadays called Cobb-Douglas production function. In its generalized form the Cobb-Douglas production function may be expressed as

$$
f\left(x_{1}, \ldots, x_{n}\right)=\gamma x_{1}^{\alpha_{1}} \ldots x_{n}^{\alpha_{n}},
$$

where $\gamma, \alpha_{1}, \ldots, \alpha_{n}>0$.

Corollary 3.1. Let $h_{1}\left(x_{1}\right), \ldots, h_{n}\left(x_{n}\right)$ be thrice differentiable functions with $h_{i}\left(x_{i}\right) \neq \gamma_{i} e^{\alpha_{i} x_{i}}$ for $i=1, \ldots, n$ and $F(u)=u$. Then the Allen matrix $M(f)$ of composite function $f(\mathbf{x})=F\left(h_{1}\left(x_{1}\right)\right.$ $\left.\times \cdots \times h_{n}\left(x_{n}\right)\right)$ is singular if and only if, up to suitable translations of $x_{1}, \ldots, x_{n}$,

$$
f(\mathbf{x})=\gamma x_{1}^{\alpha_{1}} \cdots x_{n}^{\alpha_{n}}
$$

where $\gamma_{i}, \alpha_{i}$ are some nonzero constants satisfying $\alpha_{1}+\cdots+\alpha_{n}=0$. 
Proof. Let $f=F\left(h_{1}\left(x_{1}\right) \times \cdots \times h_{n}\left(x_{n}\right)\right)$ be a twice differentiable composite function. First, let us assume that the Allen matrix $M(f)$ is singular. Under hypothesis of the theorem, it follows from Theorem 2.2 that

$$
h_{i}=\gamma_{i} x_{i}^{\alpha_{i}}, i=1, \ldots, n
$$

for some nonzero constants $\gamma_{i}, \alpha_{i}$ satisfying $\alpha_{1}+\cdots+\alpha_{n}=0$. Thus, in our case, it means that

$$
f(\mathbf{x})=\gamma x_{1}^{\alpha_{1}} \times \cdots \times x_{n}^{\alpha_{n}}
$$

Converse is easy to verify.

Remark 3.2. We have that for a generalized Cobb-douglas production function the values of $\alpha_{1}, \ldots, \alpha_{n}$ are positive constants. Hence, while Corollary 3.1 is correct in mathematical perspective, in reality such a generealized Cobb-Douglas production function does not exist.

Corollary 3.3. Let $h_{1}\left(x_{1}\right), \ldots, h_{n}\left(x_{n}\right)$ be thrice differentiable functions and let $f$ be a composite function given by

$$
f(\mathbf{x})=\ln \left(h_{1}\left(x_{1}\right) \times \cdots \times h_{n}\left(x_{n}\right)\right) .
$$

Then the Allen matrix $M(f)$ is singular if $f$ is a perfect substitute.

Proof. Let $f(\mathbf{x})=\ln \left(h_{1}\left(x_{1}\right) \times \cdots \times h_{n}\left(x_{n}\right)\right)$ be a twice differentiable composite function. If $f$ is a perfect substitute, then we write

$$
f(\mathbf{x})=\ln \left(h_{1}\left(x_{1}\right) \times \cdots \times h_{n}\left(x_{n}\right)\right)=\sum_{i=1}^{n} a_{i} x_{i},
$$

and it follows from (3.2) that

$$
h_{i}\left(x_{i}\right)=e^{a_{i} x_{i}}, i=1, \ldots, n .
$$

It means from statement (i) of Theorem 2.2 that the Allen matrix $M(f)$ is singular.

Remark 3.4. The converse of Corollary 3.3 is not always true.

\section{Some geometric interpretations of Allen determinants}

Let $(N, g)$ be a Riemannian manifold. For more detailed properties of geometric structures on Riemannian manifolds, see [3, 14]. A Riemannian connection, also called Levi-Civita connection, on the Riemannian manifold $(N, g)$ is an affine connection which is compatible with metric, i.e, $\nabla g=0$ and symmetric, i.e, $\nabla_{X} Y-\nabla_{Y} X=[X, Y]$ for any vector fields $X$ and $Y$ on $N$, where [,] is the Lie bracket.

The Riemannian curvature tensor $R$ is given in terms of $\nabla$ by

$$
R(X, Y) Z=\nabla_{X} \nabla_{Y} Z-\nabla_{Y} \nabla_{X} Z-\nabla_{[X, Y]} Z
$$


A Riemannian manifold is called a flat space if its Riemann curvature tensor vanishes identically.

Let $\sigma$ be a two dimensional subspace of the tangent space $T_{p} N$ and let $u, v \in \sigma$ be two linearly independent vectors such that $\sigma=S p(u, v)$. Then the sectional curvature of $\sigma$ at the point $p \in N$ is a real number defined by

$$
K(u, v)=K(\sigma)=\frac{g(R(u, v) v, u)}{g(u, u) g(v, v)-g(u, v)^{2}} .
$$

The Ricci tensor of a Riemannian manifold $N$ at a point $p \in N$ is defined to be the trace of the linear map $T_{p} N \longrightarrow T_{p} N$ given by

$$
w \longmapsto R(w, u) v
$$

A Riemannian manifold is called Ricci-flat if its Ricci tensor vanishes identically.

One of the B.-Y. Chen's geometric interpretations regarding $h$-homogeneous production functions from $[4,5]$ is as follows

Theorem 4.1. An $h$-homogeneous production function $f$ has flat production hypersurface if and only if either $f$ is a perfect substitute or it is of the form: $f=\left(c_{1} x_{1}+\cdots+c_{n} x_{n}\right)^{h}$, for some constants $c_{1}, \ldots, c_{n}$.

Corollary 4.2. Let $h_{1}\left(x_{1}\right), \ldots, h_{n}\left(x_{n}\right), n \geq 3$, be thrice differentiable functions and let $f$ be an $h$-homogeneous composite function given by

$$
f(\mathbf{x})=\ln \left(h_{1}\left(x_{1}\right) \times \cdots \times h_{n}\left(x_{n}\right)\right) .
$$

If the graph of $f$ is a flat space, then the Allen matrix $M(f)$ is singular.

Proof. Let $f(\mathbf{x})=\ln \left(h_{1}\left(x_{1}\right) \times \cdots \times h_{n}\left(x_{n}\right)\right)$ be an twice differentiable $h$-homogeneous composite function. Let us assume that the graph of $f$ is a flat space. Then, from Theorem 4.1, we have that $f$ is a perfect substitute. This means from Corollary 3.3 that the Allen matrix $M(f)$ is singular.

Remark 4.3. Corollary 4.2 holds also in case the graph of $f$ has vanishing sectional curvature function.

Since Ricci-flat 3-manifolds are always flat, Corollary 4.2 follows immediately

Corollary 4.4. Let $h_{1}\left(x_{1}\right), h_{2}\left(x_{2}\right), h_{3}\left(x_{3}\right)$ be thrice differentiable functions and let $f$ be an $h$-homogeneous composite function given by

$$
f(\mathbf{x})=\ln \left(h_{1}\left(x_{1}\right) \times h_{2}\left(x_{2}\right) \times h_{3}\left(x_{3}\right)\right) .
$$

If the graph of $f$ is Ricci-flat, then the Allen matrix $M(f)$ is singular. 


\section{References}

[1] R. G. Allen, J. R. Hicks, A reconsideration of the theory of value Pt. II, Economica, 1 (1934), 196-219.

[2] K. J. Arrow, H. B. Chenery, B. S. Minhas, R. M. Solow, Capital-labor substitution and economic efficiency, Rev. Econom. Stat., 43 (1961), 225-250.

[3] B.-Y. Chen, Geometry of submanifolds, M. Dekker, New York 1973.

[4] B.-Y. Chen, On some geometric properties of h-homogeneous production function in microeconomics, Kragujevac J. Math., 35 (2011), 343-357.

[5] B.-Y. Chen, G. E. Vîlcu, Geometric classifications of homogeneous production functions, Appl. Math. Comput., 225 (2013), 345-351.

[6] B.-Y. Chen, Classification of h-homogeneous production functions with constant elasticity of substitution, Tamkang J. Math., 43 (2012), 321-328.

[7] B.-Y. Chen, On some geometric properties of quasi-sum production models, J. Math. Anal. Appl., 392 (2012), 192-199.

[8] B.-Y. Chen, Geometry of quasi-sum production functions with constant elasticity of substitution property, J. Adv. Math. Stud., 5(2) (2012), 90-97.

[9] B.-Y. Chen, Classification of homothetic functions with constant elasticity of substitution and its geometric applications, Int. Electron. J. Geom., 5(2) (2012), 67-78.

[10] B.-Y. Chen, An explicit formula of Hessian determinants of composite functions and its applications, Kragujevac J. Math. 36 (2012), 27-39.

[11] B.-Y. Chen, A note on homogeneous production models, Kragujevac J. Math., 36 (2012), 41-43.

[12] B.-Y. Chen, Solutions to homogeneous Monge-Ampere equations of homothetic functions and their applications to production models in ecenomics, J. Math. Anal. Appl., 411 (2014), 223-229.

[13] C. W. Cobb and P. H. Douglas, A theory of production, Amer. Econom. Rev., 18 (1928), 139-165.

[14] P. M. do Carmo, Riemannian Geometry, Birkhäuser, Boston, 1992.

[15] C. A. Ioan, Applications of the space differential geometry at the study of production functions, EuroEconomica, 18 (2007), 30-38.

[16] L. Losonczi, Production functions having the CES property, Acta Math. Acad. Paedagog. Nyhái. (N.S.), 26 (2010), 113-125.

[17] A. Mihai, M. Sandu, The use of the h-homogeneous production function in microeconomics. Modelling challenges, Revista Economica, 1 (2012), 465-472.

[18] A. Mihai, A. Olteanu, Applied geometry in microeconomics. Recent developments, Land Reclamation, Earth Observation \& Surveying, Enverionmental Enginnering, II (2013), 159-166,

[19] S. K. Mishra, A brief history of production functions, IUP J. Manage. Econom., 8(2010), 6-34.

[20] H. Uzawa, Production functions with constant elasticities of substitution, The Review of Economic Studies, 29 (1962), 291-299.

[21] G. E. Vilcu, A geometric perspective on the generalized Cobb-Douglas production functions, Appl. Math. Lett., 24 (2011), 777-783.

[22] A. D. Vilcu and G. E. Vilcu, On some geometric properties of the generalized CES production functions, Appl. Math. Comput., 218 (2011), 124-129.

[23] A. D. Vilcu and G. E. Vilcu, On homogeneous production functions with proportional marginal rate of substitution, Mathematical Problems in Engineering (2013), doi.10.1155.

Department of Mathematics, Faculty of Science, Firat University, 23200, Elazig, Turkey.

E-mail: meaydin@firat.edu.tr

E-mail: mergut@firat.edu.tr 